\title{
Estimation of the surface area-to-volume ratios of litter components of the Brazilian rainforest and their impact on litter fire rate of spread and flammability
}

\author{
Paulo Bufacchi ${ }^{1}$ D . José Carlos Santos ${ }^{2} \cdot$ João Andrade de Carvalho Jr. $^{3} \cdot$ Guenther Carlos Krieger Filho $^{1}$
}

Received: 29 April 2019 / Accepted: 20 March 2020 / Published online: 30 April 2020

(c) The Brazilian Society of Mechanical Sciences and Engineering 2020

\begin{abstract}
The surface-area-to-volume ratio (SAV) is a significant parameter in vegetation description, although different researchers have presented diverging estimates for forest litter. However, considering litter as a unique entity and disregarding the SAV of its components provides a misleading fire rate of spread. Undoubtedly, the leaves are more relevant than the twigs for the rate of spread of surface fires, as the former has a higher SAV. Therefore, based on the proven hyperdominance of tree species in the Brazilian rainforest, this work presents an accurate method to estimate the SAV. Furthermore, it demonstrates the critical role played by the SAV in the litter flammability and fire rate of spread assessments and shows that the leaf moisture content does not influence its SAV. Besides, comparing SAV data using the methodology presented herein with other authors' assessments, the results differ by up to $49 \%$. Finally, using the Brazilian rainforest litter in the methodologies of two distinct authors showed a difference of at least $36 \%$ in the results. The primary outcome of this study is the estimation of the SAV of leaves and thin, medium, and thick twigs, representative of the Brazilian rainforest, at $12,680 \mathrm{~m}^{-1}, 1360 \mathrm{~m}^{-1}$, $620 \mathrm{~m}^{-1}$, and $310 \mathrm{~m}^{-1}$, respectively. The estimated SAV values are essential input data to numerical simulation models, and for comparison purposes with other authors' work, the litter SAV is $8460 \mathrm{~m}^{-1}$.
\end{abstract}

Keywords Surface-area-to-volume $\cdot$ Litter $\cdot$ Brazilian rainforest $\cdot$ Flammability $\cdot$ Rate of spread

\section{Introduction}

Technical Editor: Mario Eduardo Santos Martins, Ph.D.

Paulo Bufacchi

paulo.bufacchi@usp.br; paulo_bufacchi@hotmail.com

José Carlos Santos

jose.santos@inpe.br

João Andrade de Carvalho Jr.

joao@feg.unesp.br

Guenther Carlos Krieger Filho

guenther@usp.br

1 Laboratory of Thermal and Environmental Engineering, Department of Mechanical Engineering, School

of Engineering of the University of São Paulo, Av. Prof. Mello de Morais 2231, São Paulo, SP 05508-030, Brazil

2 Combustion and Propulsion Associated Laboratory, National Institute for Space Research (INPE), Cachoeira Paulista, SP 12630-000, Brazil

3 Department of Energy, São Paulo State University (UNESP), Guaratinguetá, SP 12516-410, Brazil
The surface-area-to-volume ratio (SAV) is undoubtedly the most significant factor in determining how size influences biological design [1]. The surface area represents the interface between litter (leaves and twigs) and its environment and is an indication of the intensity of the exchange of nutrients and other substances. Volume, on the other hand, holds plant structure and internal processes [1]. Therefore, both surface area and volume are essential to study the mechanisms of substances exchange in leaves. However, they do not keep a simple proportionality with each other, as the surface area is proportional to the square of lengths, and the volume is to the cube. It means that changing both variables at the same time may result in modifying leaf shape [1] and, for the litter, average SAV and fire rate of spread (ROS).

SAV is an essential parameter in vegetation description in fire simulations, as it strongly influences vegetation flammability [2-4]. Fuels that present a higher SAV have faster moisture loss, which would decrease time to ignition $[5,6]$ and increase ROS. Furthermore, environmental variables 
such as relative humidity and air temperature have a great influence on the consumption of fuels with higher SAV, while smoldering combustion is less dominant in high SAV fuels [7].

In Rothermel's fire spread model [8], SAV is one of the basic parameters and is a relevant input variable for the fire modeling systems BehavePlus [9] and WFDS (WildlandUrban Fire Dynamics Simulator) [10]. In WFDS equations, SAV is present in the drag force the vegetation exerts on the airflow, convective and radiative heat transfers, moisture evaporation, and vegetation volatilization [11, 12]. Using WFDS, Bufacchi et al. [12] and Bufacchi [13] showed that ROS decreases as the vegetation SAV decreases. Therefore, for an accurate ROS assessment, which is essential in fire management [14], a precise SAV assessment is imperative.

Litter is an important fuel source in forest surface fires, and its amount on the forest floor depends on the balance between litter production and decomposition [15]. In general, forest litter consists mainly of leaves and twigs from several different tree species fallen onto the ground (Oi layer). As a result, many researchers presented diverse SAV estimates for different vegetation types, considering litter as a single entity and estimating only one SAV value. However, disregarding the SAV of individual litter components such as leaves and twigs provides a misleading fire rate of spread, as it has a strong influence from SAV. Groundbreaking studies in this field, such as the one from Rothermel and Anderson [5], present SAV as high as $9154 \mathrm{~m}^{-1}$ for needles and as low as $315 \mathrm{~m}^{-1}$ for sticks. Later, Rothermel [8] presented an SAV of $8202 \mathrm{~m}^{-1}$ for fine hardwood litter, although no further information explains how they obtained the SAV values. BehavePlus [9] uses three loads of broadleaf litter: a low load (t12), moderate load (t16), and very high load (t19). According to Scott and Burgan [16], characteristic SAV is $5925 \mathrm{~m}^{-1}$ for tl 2 fuel type, $6352 \mathrm{~m}^{-1}$ for t16, and $5686 \mathrm{~m}^{-1}$ for t19. Live herbaceous and live woody classes had values assigned for each draft fuel model, although BehavePlus allows users to input their SAV for ad-hoc calculations. In the Brazilian rainforest, however, the number of different tree species and their spatial distribution prevents the litter qualification and quantification in terms of plant species. Therefore, characterization of the forest litter as bulk is mandatory, and its properties are average values among local species.

Brown [17], Fernandes and Rego [2], Mauseth [18], and Hachmi et al. [19] proposed different methods of evaluating SAV. Measuring thickness and using blueprint outlines of hardwood leaves such as Aspen (Populus tremuloides Michx.) and Eucalyptus (Eucalyptus obliqua L'Herit.), Brown [17] determined their SAV as $13,980 \mathrm{~m}^{-1}$ and $6180 \mathrm{~m}^{-1}$, respectively. However, it is not clear if there was a measurement of the lamina only or if the analyses included midvein and veins as well. Differently, Fernandes and Rego
[2] used a water immersion technique to determine the SAV of five important species of Northern Portugal shrubland and forest understory, which ranged from 3760 to $9400 \mathrm{~m}^{-1}$. Additionally, Mauseth [18] studied succulent shoots and ordinary flat non-succulent leaves, but only reported the SAV for succulent shoots. Finally, Hachmi et al. [19] developed a geometric method for SAV estimation, based on the perimeter-to-area ratio concept, to measure the dimension of leaves, twigs, and needles. The SAV of the leaves from Moroccan trees and shrubs species ranged from 4464 to $11,224 \mathrm{~m}^{-1}$. The common point of the works mentioned above is that they estimate SAV per leaf species and not for the litter. Although the previous works are necessary to understand the SAV behavior of specific species, there is a need to have a different methodology to study forest litter, as they comprise diverse unknown leaf species.

The main objective of this work is to introduce a method to estimate the SAV of the Brazilian rainforest litter components while considering the challenges leaf morphology present to computing SAV. Moreover, it recommends an uncomplicated approach to quickly assess leaves SAV, besides demonstrating the critical role SAV plays in litter flammability and ROS assessments and evaluating the significance of leaf moisture content to the SAV. To the authors' knowledge, no works studied the SAV of the Brazilian rainforest litter components, the impact of the SAV value on ROS and flammability, and the influence of leaves moisture content on SAV.

\section{Materials and method}

\subsection{Litter collection}

Litter collection occurred in a site of 2.5 ha in the State of Rondonia, northern Brazil, which represents the Brazilian rainforest biome. This on-site work took place by the end of the dry season, centered on coordinates $08^{\circ} 37^{\prime} 58.2^{\prime \prime} \mathrm{S}$ $063^{\circ} 11^{\prime} 51.7^{\prime \prime} \mathrm{W}$ (Fig. 1). A square frame measuring $0.5 \mathrm{~m}$ by $0.5 \mathrm{~m}$ delimits the region to collect the litter (dead leaves and twigs), composing a sample. Each sample had the leaves and branches placed in different bags, which gave a total of fifty-six samples collected. Finally, sample collection ended up sealing them and sending them to the Laboratory of Environmental and Thermal Engineering at the University of São Paulo-Brazil.

Although the Brazilian rainforest extends for thousands of $\mathrm{km}^{2}$, covering about 16,000 species, only 227 of them represent half of the trees in the biome [20]. Therefore, choosing a low-diversity area where hyperdominant trees percentage are higher, such as the southern Brazilian rainforest [20], would provide a very representative litter. The selected area has approximately $70 \%$ of trees belonging to 

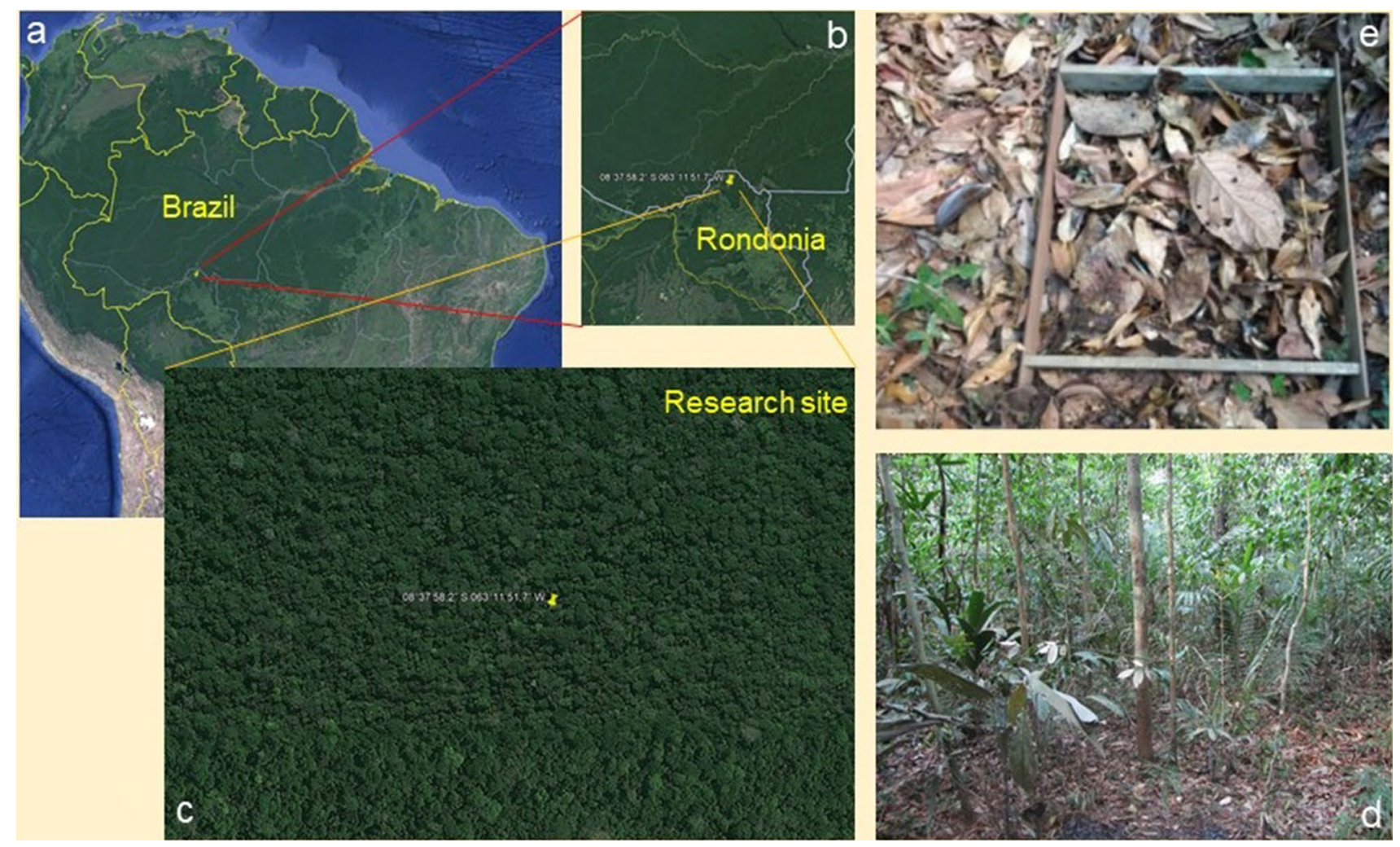

Fig. 1 Overview of the location where the material was collected. a overview of the site in the Brazilian rainforest (dark green). b State of Rondonia, where the material was collected. $\mathbf{c}$ Closer view of the

this hyperdominant group [20]. Undoubtedly, it is correct to state that the collected litter belongs to an area that assures results to be representative of the biome.

\subsection{SAV theory and leaf morphology}

The SAV estimate for a flat leaf is the ratio of the total surface area (top, bottom, and side) to the volume. For flat leaves, the SAV estimate can use the equation:

$\mathrm{SAV}=\frac{S}{V}=\frac{2 A+P t}{A t}$

where $S$ is the total surface area of the leaf, $V$ is the volume, $A$ is the top or bottom surface area, $P$ is the perimeter, and $t$ is the thickness. The challenge in this equation is to determine the surface areas, as the leaves in the litter of the Brazilian rainforest are usually decomposed or fragmented (Fig. 2). Moreover, as the leaf area times its thickness results in the leaf volume, a simplification that eliminates the area parameter is critical.

The lamina has a flat geometry, so it is reasonable to suppose that $2 A \gg P t$, which after simplifications, provides an SAV of $2 / t$. Therefore, the obstacle becomes to accurately measure the thickness of the leaves, which makes it
Rainforest and the collection site center. d View of the Rainforest from the floor in one of the sites. e Detail of the frame used to collect litter. Figures a, b and c are from Google Earth and DigitalGlobe

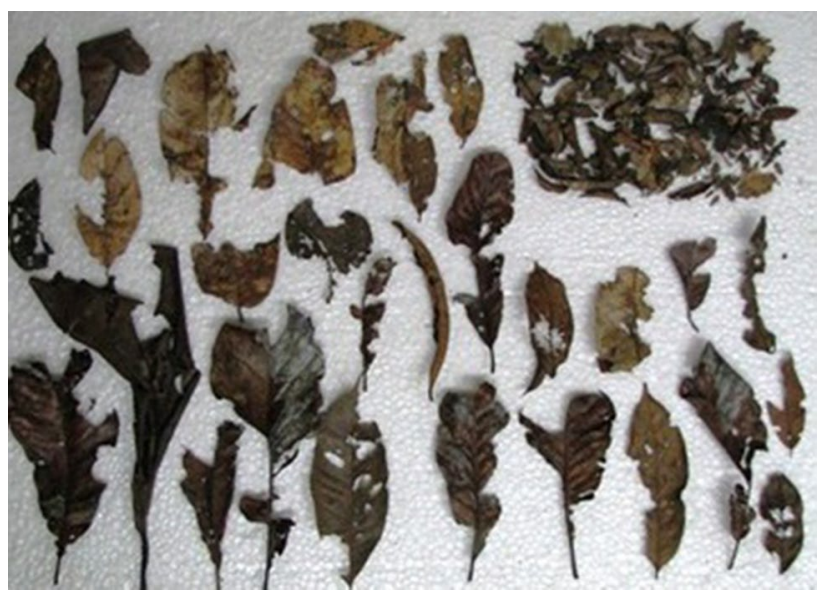

Fig. 2 Leaves collected in the Brazilian rainforest litter. Leaf decomposition or fragmentation can be significant, regardless of leaf size. This creates a difficulty for surface area, perimeter and thickness measurements for SAV estimation

necessary to understand their morphology. Regardless of leaf size, the observation of leaves collected in the Brazilian rainforest litter reveals that there is a general structure: midvein, veins, and lamina. The midvein is the thickest 
component of the leaf, and its thickness varies significantly from end to end of the leaf length. The veins are thinner than the midvein and, although their thickness varies along their length, this variation is proportionally smaller than the midvein variation. Lastly, the lamina is the thinnest element, and its thickness deviates little from point-to-point. Therefore, a procedure to estimate the leaf thickness needs to be in place to minimize discrepancies in SAV estimation and, at the same time, to consider the three elements of the leaf structure.

Veins, midveins, and twigs are approximately cylindrical, and SAV estimation can thus use the following equation

$\mathrm{SAV}=\frac{S}{V}=\frac{2 A_{\mathrm{e}}+P L}{A_{\mathrm{e}} L}$

where $S, V, A_{\mathrm{e}}, P$, and $L$ are the cylinder total surface area, volume, top-bottom surface area and perimeter, and length. Recognizing that the cylinder length is considerably greater than its diameter, the SAV is equivalent to $4 / d$ for veins, midveins, and twigs.

\subsection{Planning and methodology of the measurements}

As all leaves have a midvein, veins, and lamina, which have different shapes and thicknesses, the methodology chosen provides a comprehensive estimation of the SAV, considering the contribution of all three components. Therefore, it is inevitable to analyze an SAV for each leaf component, followed by a weighed average based on the volume or mass of the individual components to form the leaf SAV. Assuming that the density of the leaf is constant, i.e., it is the same for midvein, veins, and lamina, both weighing methods give the same results. Moreover, the weighed average based on the volume of midvein and veins would require a challenging and imprecise procedure, as the diameter is not constant everywhere. Consequently, the weighed average based on the mass is a natural choice, as detailed in Appendix Figs. 7 and 8 , which show the procedures used to estimate leaves and twigs SAV. Additionally, these figures show that SAV determination takes into account the average of the averages, where the final average value represents data from all the sites studied. Finally, the Central Limit Theorem states that the distribution of means is always normally distributed, regardless of the original distribution of the independent variables. Furthermore, the average of the averages must converge to the true mean value of the population.

The leaf lamina is the most sensitive part of the leaf, so measurements started with it to avoid over-manipulating it. Measuring the lamina with care not to include the veins, avoided reporting a thickness that would be higher than it is. Measurements used a Mitutoyo LCD caliper $0.01 \mathrm{~mm}$ resolution and repeatability, done three times for each thickness. Initially, ten measurements on the lamina, five on each side of the leaf midvein, confirmed the average thickness. Next, it took ten measurements to validate the midvein diameter along the leaf length, the same number for the veins, being five on each side of the leaf, from the midvein to the leaf edge. As a result, ninety measurements supported each leaf thickness.

The next step was to calculate the weighed average, and the dissection of the leaves separated the midvein, veins, and lamina for weighing, using a Marte model AD500 precision weight balance $0.001 \mathrm{~g}$ sensitivity and reproducibility. The statistical parameters average, standard deviation, and coefficient of variation characterize the weight for each leaf component. The average weight of each of the three components supported the determination of its percentage in the total mass, based on eighty-four different measured, dissected, and weighed leaves. Finally, the SAV evaluation of each leaf took one hundred and five measurements, in a total of 8820 for the litter SAV, with a similar procedure estimating the SAV for twigs.

\subsection{SAV assessment using methodologies from other authors}

Measurements designed to assess the work of Hachmi et al. [19] tested whether the proposed methodology could provide correct SAV data for the leaves of the Brazilian rainforest. A selection of different leaves in terms of species and sizes examined their method, whose central proposition is that every leaf has an elliptical cross-section. Therefore, cutting the selected leaves in half concerning their length, and each part in half again, provides six measurements at the cutting edges of each of the parts, three on each side of the midvein. Measurements used a Mitutoyo LCD caliper $0.01 \mathrm{~mm}$ resolution and repeatability, done three times for each thickness.

With the same previous purpose, measurements evaluated the technique proposed by Fernandes and Rego [2], which uses water immersion to estimate the SAV. The SAV value results from the leaf weight before and after immersion, along with the leaf density and the thickness of the adsorbed water pellicle, which is a theoretical average value. The leaf immersion in a container with water changes the liquid height, measured by a scale. In this case, measurements used a Marte model AD500 precision weight balance $0.001 \mathrm{~g}$ sensitivity and reproducibility and a scale, done three times for each thickness.

\subsection{Impact of leaf moisture content on SAV assessment}

An experiment conducted with Carapa guianensis and Brownea grandiceps leaves verified if the leaf moisture 
content influences the SAV. These leaf species, widely found in the Brazilian rainforest biome, originated from their trees and not from the litter.

Each leaf had its length, width, midvein, veins, and lamina thicknesses measured in triplicate. The measurements taken in the vein and midveins were in three positions on each leaf, while the thickness of the lamina was in five places. A marker identified all the measuring positions in each leaf to assure thickness, length, and width assessments in the same location every time. Measurements started on the day of leaves collection, which continued on days 2, 3, 4, 10 , and 14, always at the same time. All leaves remained in a laboratory environment and dried under local conditions, except on the 14th day when they dried in an oven for $72 \mathrm{~h}$ at $60{ }^{\circ} \mathrm{C}$.

\section{Results and discussion}

\subsection{Litter components thicknesses and SAV estimation}

The measurement results validate that the leaf lateral area is considerably less than the top and bottom areas, due to its small thickness. Disregarding the leaf side area causes an impact on SAV estimation that is less than $1 \%$. Therefore, there is a rather small error when considering leaves SAV equal to two divided by its thickness $(2 / t)$. Thicknesses of lamina, veins, and midvein for large, medium, and small leaves, as presented in Fig. 3, raise the question if a single component can represent its thickness.

Although lamina thickness is practically constant in every part of a single leaf, its average value is higher in larger leaves, yet the difference is not as high as in veins and midvein. Larger leaves noticeably have thicker veins and midvein. As large, medium, and small size leaves have similar lamina thickness, medium and large size leaves have proportionally larger veins and midvein to provide a more robust structure to support the lamina. On average, lamina thicknesses vary from 0.14 to $0.20 \mathrm{~mm}$ from small to large leaves, while veins vary from 0.20 to $0.43 \mathrm{~mm}$ and midvein from 0.48 to $1.06 \mathrm{~mm}$. However, as leaf SAV is not directly related to length or width, small leaves and large ones have the same SAV when they have equal thickness. Moreover, the outliers are more pronounced in veins and midveins due to larger variations in their diameters, and they occur on large and medium-size leaves only.

Twigs thicknesses, shown in Fig. 4, have three categories: thin (up to $4 \mathrm{~mm}$ ), medium (from 4 to $10 \mathrm{~mm}$ ) and thick (10 $\mathrm{mm}$ and up) sizes. The average thicknesses of twigs are much higher than those of the lamina, veins, and midvein. The outliers in Fig. 4 are clearly shown only for thick twigs, due to wider fluctuations in their dimensions.

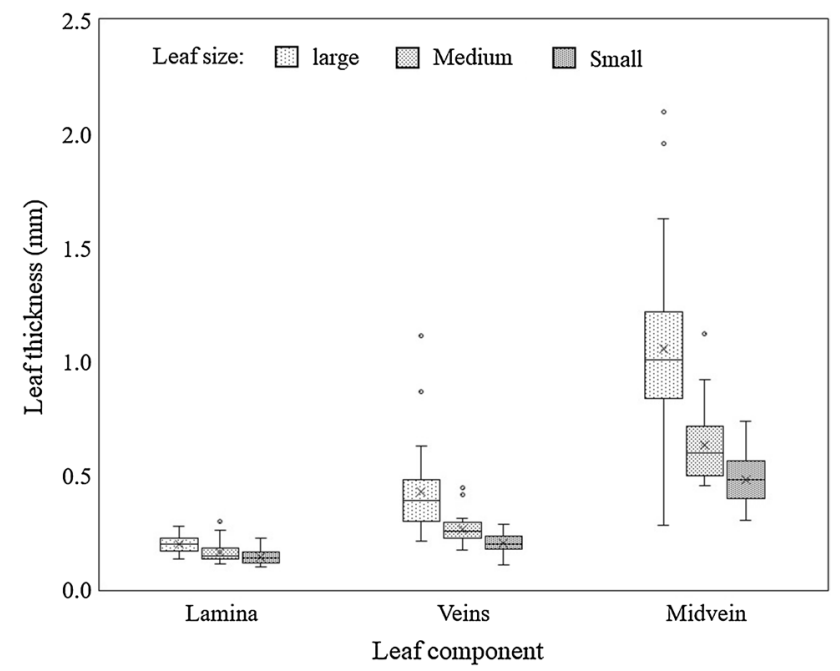

Fig. 3 Thickness for lamina, veins and midvein, separated by large, medium and small leaves. Boxplots show the median (horizontal line), mean value ( $x$ ), upper (third) and lower (first) quartiles in the box and separated by the median, and upper and lower variability outside the quartiles depicted with lines (whiskers). Points outside whiskers are outliers. A point is an outlier when its numerical value is greater than 1.5 times the difference between the third and the first quartiles

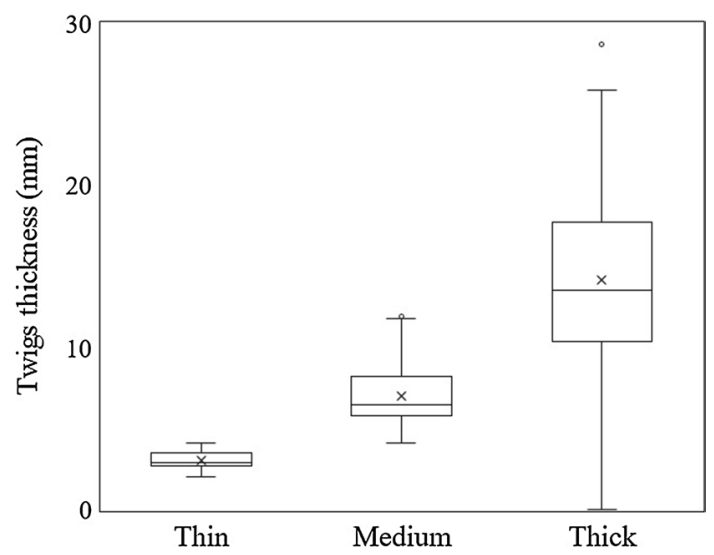

Fig. 4 Thickness for twigs (thin, medium and large). Measurement variation is higher for thick twigs, as their maximum thickness is not limited. Boxplots show the median (horizontal line), mean value $(x)$, upper (third) and lower (first) quartiles in the box and separated by the median, and upper and lower variability outside the quartiles depicted with lines (whiskers). Points outside whiskers are outliers. A point is an outlier when its numerical value is greater than 1.5 times the difference between the third and the first quartiles

The SAV estimate based on the thickness measurement, shown in Fig. 5, exhibits a lamina SAV that is less than the veins SAV, which may be inconsistent at first. Although the average lamina thickness for all measured leaves is $0.17 \mathrm{~mm}$, the veins that have a larger average diameter, $0.30 \mathrm{~mm}$, have a higher SAV because their calculated value for the 


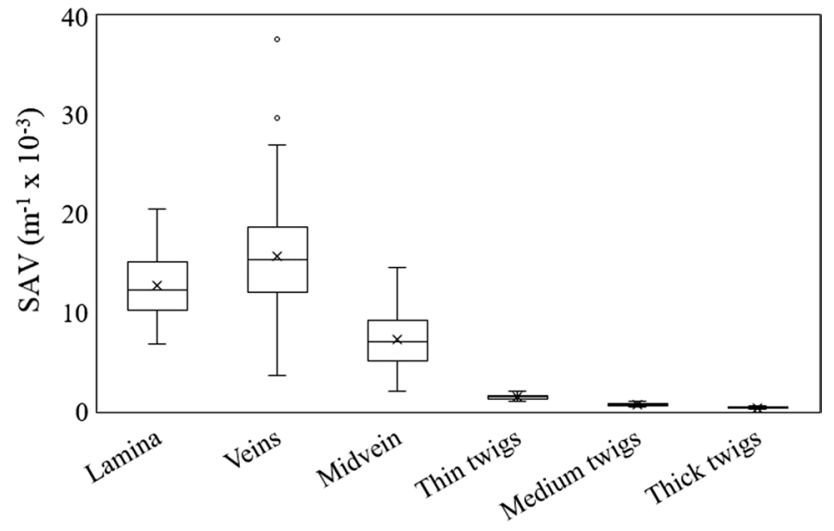

Fig. 5 SAV separated by lamina, veins, midvein and twigs (thin, medium, and thick). As SAV equation has thickness in the denominator, leaves components have the greatest SAV. Boxplots show the median (horizontal line), mean value $(x)$, upper (third) and lower (first) quartiles in the box and separated by the median, and upper and lower variability outside the quartiles depicted with lines (whiskers). Points outside whiskers are outliers. A point is an outlier when its numerical value is greater than 1.5 times the difference between the third and the first quartiles

lamina and veins is $2 / t$ and $4 / d$, respectively. The average leaf SAV weighted by the leaf mass is $12,680 \mathrm{~m}^{-1}$, which is a combination of $80 \%$ of the lamina, $7 \%$ of veins, and $14 \%$ of midvein in mass. This average is closer to lamina SAV $\left(12,710 \mathrm{~m}^{-1}\right)$, as veins SAV $\left(15,710 \mathrm{~m}^{-1}\right)$ and midvein SAV $\left(7190 \mathrm{~m}^{-1}\right)$ counterbalance each other around the lamina SAV. The difference between leaf and lamina SAV is only $0.2 \%$, which is a very relevant conclusion on leaf SAV and to the authors' knowledge, no previous work has demonstrated this finding.

Regarding twigs, the SAV for thick, medium, and thin diameters is $310 \mathrm{~m}^{-1}, 620 \mathrm{~m}^{-1}$, and $1360 \mathrm{~m}^{-1}$, respectively. In numerical simulation codes, to correctly assess ROS, it is better to create elements with separate SAV. Recognizing that the average SAV of thin twigs is over nine times smaller than leaves SAV, the leaves contribution to litter flammability and ROS is significantly more important than that from twigs, which is also a very relevant proven result of this work.

Following the methodology for leaves and twigs SAV estimation detailed in Figs. 7 and 8, an average litter SAV value representative of the Brazilian rainforest estimate is $8460 \mathrm{~m}^{-1}$. This value should be used only for purposes of comparison with other authors, but not in numerical simulations, as it will misrepresent the fire rate of spread.

\subsection{SAV variation and its effect on litter flammability and ROS assessment}

Many modeled fire processes use SAV as explained in Sect. 1, but an incorrect value for SAV can have an adverse

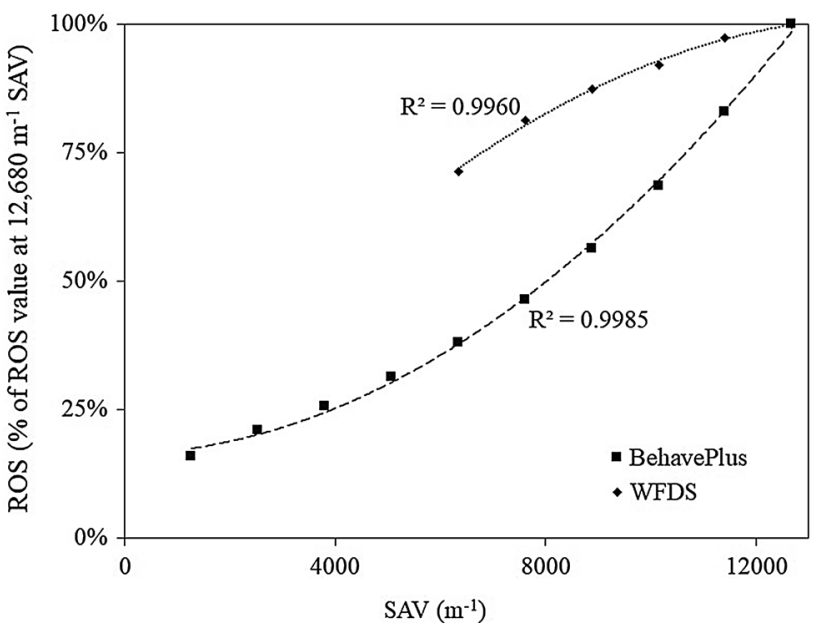

Fig. 6 Influence of SAV variation on litter flammability and ROS assessment using BehavePlus and WFDS numerical simulation. Second degree polynomials adjust the calculated fire rate of spread well. WFDS shows a fire spread scenario when SAV is as low as $50 \%$ of the estimated SAV $\left(12,680 \mathrm{~m}^{-1}\right)$. Conversely, BehavePlus show a fire spread scenario for all the tested SAV values

impact on calculated litter flammability and ROS, as shown in Fig. 6. WFDS and BehavePlus numerically simulated the influence of SAV on litter flammability and ROS assessments. A second-order polynomial trend line connects WFDS and BehavePlus data points in Fig. 6 and fits the simulated data well ( $R^{2}$ of 0.9960 and 0.9985 , respectively). In the graph, ROS is a percentage referring to a maximum value (reference value) obtained through numerical simulation using the maximum leaf SAV value of $12,680 \mathrm{~m}^{-1}$. The other chosen SAV values decrease by a rate equal to ten percentage points, starting with the maximum SAV value. Both WFDS and BehavePlus data show a decrease in ROS as SAV also decreases, but with different curve shapes. BehavePlus results show that fire spreads until SAV values reach $10 \%$ of its maximum value, while WFDS shows a no-spread fire scenario when SAV reaches $40 \%$ of its maximum value. The spread and no-spread fire scenarios present a significant impact on fire management and substantiate the requirement of evaluating a correct SAV of the fuel.

BehavePlus simulation varied 1-h SAV from 12,680 to $1268 \mathrm{~m}^{-1}$ in a $10 \%$ decrement of the highest SAV. The other parameters remained constant: 1-h Fuel Load at 0.8946, Fuel Bed Depth at $3.6 \mathrm{~cm}$, Dead Fuel Moisture of Extinction at $40 \%$, Dead Fuel Heat Content at 20,000 kJ/kg, and Dead Fuel Moisture at 26\%. The other parameters were not used and left blank or zero.

WFDS simulation employed a mesh size defined by a mesh independence analysis while using a numerical ignition that did not influence the estimated ROS. WFDS variable veg_sv ranged from 12,680 to $5072 \mathrm{~m}^{-1}$ in $10 \%$ decrement of the estimated SAV. Fire did not spread for SAV 
value of $5072 \mathrm{~m}^{-1}$. Other variables used are: heat_of_combustion $20,000 \mathrm{~kJ} \mathrm{~kg}^{-1}$, veg_initial_temperature $28.7^{\circ} \mathrm{C}$, veg_moisture 0.26 , veg_char_fraction 0.06 , veg_ash_fraction 0.0375 , veg_density $435 \mathrm{~kg} \mathrm{~m}^{-3}$, veg_bulk_density (fuel bulk density) $19.93 \mathrm{~kg} \mathrm{~m}^{-3}$, veg_degradation according to 'Arrhenius' equation, and veg_char_oxidation 'true'.

Wildland fires, either surface or crown fires, rely on adequate ROS assessments to accurately plan and execute fire suppression, provide required safety to firefighters and to issue timely warnings to preserve life and property [14]. The efficient assessment of the fire rate of spread is dependent on correct surface-area-to-volume ratio evaluation.

\subsection{SAV assessment using methodologies from other authors}

The central premise of Hachmi et al. [19] work is that the cross-section of any forest fuel has the shape of an ellipse. Therefore, the eccentricity e describing the shape variation throughout its width is an important variable and, among the four classes defined for $\mathrm{e}$, the leaves range was $0.35 \leq e<1$. The analysis of the Brazilian rainforest leaves revealed that this premise is not adequate, as the more than eleven thousand thickness measurements carried out in this work show that the leaf cross-section is very similar to a rectangle $(e \cong 1)$. Even so, an attempt to use their equations to estimate Brazilian rainforest litter leaves SAV would overestimate the values by approximately $36 \%$ when compared to the methodology presented herein.

Using Fernandes and Rego [2] model equation and reproducing their experiment with Brazilian rainforest litter, we found a widely underestimated SAV, as also reported by Hachmi et al. [19]. Unfortunately, among the leaves used by Fernandes and Rego [2] to validate their model, there were not leaves found in the Brazilian rainforest, which may explain the difference found using their methodology.

\subsection{Impact of leaf moisture content on SAV estimation}

Carapa guianensis and Brownea grandiceps leaves have different behavior when drying. From the collection of the leaves to the last experiment when leaves were ovendried, Brownea grandiceps length and width changed very little, the same happening to its lamina, veins, and midvein thicknesses. Although the changes in Carapa guianensis length, width, and lamina were very small, the changes in veins and midvein thickness were more significant. In summary, there is a very small decrease in SAV for Carapa guianensis (2.1\%) and a very small increase in SAV for Brownea grandiceps $(1.8 \%)$ between the initial and the final experimental conditions. The experimental conditions are extreme situations, as the leaves final moisture content in laboratory conditions is much lower than the moisture content found inside the forest. Also, green leaves moisture contents are similar to litter moisture content. In Brazilian rainforest conditions, changes in SAV are smaller than the numbers presented for completely dry leaves. It is then correct to say that there is no impact on SAV estimation because of leaf moisture content changes.

\subsection{Comparison of SAV values among authors}

The authors referenced herein and the SAV estimated in their studies for litter [8, 16] and leaves [2, 17, 19] show in Table 1, while the last six lines present the data obtained herein. Populus tremuloides Michx. SAV estimated by Brown [17] is a little over 10\% higher than the SAV estimated herein for litter leaves, which is approximately $43 \%$ higher than the Quercus suber estimated by Hachmi et al. [19]. Both Populus tremuloides Michx. and Quercus suber are broadleaves, as well as the Brazilian

Table 1 Comparison among SAV $\left(\mathrm{m}^{-1}\right)$ estimated by different researchers for diverse litter or leaf species, where the last three lines refer to data estimated herein

\begin{tabular}{|c|c|c|c|c|c|c|}
\hline Litter/leaf type & Rothermel [8] & $\begin{array}{l}\text { Scott and } \\
\text { Burgan [16] }\end{array}$ & Brown [17] & $\begin{array}{l}\text { Fernandes and } \\
\text { Rego [2] }\end{array}$ & $\begin{array}{l}\text { Hachmi } \\
\text { et al. [19] }\end{array}$ & This work \\
\hline Fine hardwood litter & 8202 & & & & & \\
\hline Broadleaf litter: low load (t12) & & 5925 & & & & \\
\hline Broadleaf litter: moderate load (t16) & & 6352 & & & & \\
\hline Broadleaf litter: very high load (t19) & & 5686 & & & & \\
\hline Aspen (Populus tremuloides Michx.) leaves & & & 13,980 & & & \\
\hline Eucalyptus (Eucalyptus obliqua L'Herit.) leaves & & & 6180 & 5690 & 6922 & \\
\hline Quercus suber L. & & & & & 8887 & \\
\hline Brazilian Amazon litter (leaves only) & & & & & & 12,680 \\
\hline Brazilian Amazon litter (twigs only) & & & & & & 770 \\
\hline Brazilian Amazon litter (leaves and twigs) & & & & & & 8460 \\
\hline
\end{tabular}


rainforest leaves. ROS based on BehavePlus calculations for these two leaves is, respectively, 1.16 and 0.57 times the ROS assessment with the SAV herein. Examining the litter data, SAV estimated in this work is a little over 3\% higher than the value proposed by Rothermel [8] for fine hardwood litter. In turn, it is approximately $33-49 \%$ higher than the values for different loads presented by Scott and Burgan [16]. To further strengthen the importance of a correct SAV assessment, BehavePlus simulated ROS using data from Rothermel [8] and Scott and Burgan [16]. Using the fine hardwood litter SAV from Rothermel [8], ROS is almost $52 \%$ of the calculated ROS using this work data (leaves and twigs separately). This result is very relevant, because although the difference in litter SAV is small (3\%), ROS is significantly different, because litter is composed of materials that have very diverse SAV. Therefore, the use of a single value for litter SAV in ROS calculation does not provide accurate results, whereas using separate SAV for each litter component does. Finally, estimated ROS using broadleaf litter low load (t12) from Scott and Burgan [16] shows a difference that is a little over 35\% of the value using the work herein. It is worth noting that t12 value for SAV is very close to WFDS flammability limiting SAV, where litter would not ignite and there would be a no-spread fire scenario.

\subsection{Proposal of a simple method to obtain Brazilian rainforest leaves SAV}

The procedure detailed herein to estimate the SAV of Brazilian rainforest litter produced a result as accurate as possible. However, the procedure is time-consuming, and an ad-hoc SAV assessment will need a simple methodology. On average, the lamina is the most representative component in a leaf structure, accounting for $80 \%$ of its mass, and veins and midvein SAV practically counterbalance each other. Therefore, it seems straightforward to consider the lamina SAV as the leaves SAV, since the difference between them is around $0.2 \%$.

\section{Conclusion}

This work presents a methodology to estimate the SAV of the Brazilian rainforest litter, which has many leaf species. It also provides an average SAV value for leaves and thin, medium, and thick twigs individually. It then shows a simplified method to estimate leaves SAV based on the leaf lamina.
The SAV estimation of leaves and thin, medium, and thick twigs, representative of the Brazilian rainforest, are $12,680 \mathrm{~m}^{-1}, 1360 \mathrm{~m}^{-1}, 620 \mathrm{~m}^{-1}$, and $310 \mathrm{~m}^{-1}$, respectively. The average leaf lamina SAV is very close to the average leaves SAV, a difference of $0.2 \%$ only. For comparison purposes with other authors' work, the estimate of the litter SAV is $8460 \mathrm{~m}^{-1}$. These SAV average values are relevant as input data to numerical simulation models such as BehavePlus and WFDS.

Numerical simulations using WFDS and BehavePlus investigated the influence of SAV variation on litter flammability and ROS assessment. According to WFDS, as the SAV reaches $40 \%$ of its maximum value, there is a no-spread fire scenario. This SAV variation presents a significant impact in fire spread scenarios and substantiates the requirement of evaluating a correct SAV for the studied fuel.

This work also evaluates the influence of leaf moisture content on the SAV, showing that variations in length, width, and thicknesses do not influence the SAV. Therefore, it is correct to say that there is no impact on SAV estimation because of leaf moisture content changes.

Comparisons between this work and other researchers' data show that the differences in SAV affect flammability and ROS, and reinforces the importance of a precise determination of SAV. It also shows that although the difference in litter SAV may be small, ROS evaluation may be significantly different, because the litter has materials with very diverse SAV.

The Brazilian rainforest leaves collected for this work served as input to two other methodologies [2, 19], for comparison purposes with this work. SAV values using those methodologies differ significantly from the ones presented herein.

Acknowledgements Partial funding for this work was received from the São Paulo Research Foundation (FAPESP) Programs under Projects 2008/04490-4 and 2011/20679-2.

\section{Compliance with ethical standards}

Conflict of interest The authors declare that they have no conflict of interest.

\section{Appendix}

This appendix presents detailed information about the procedures used to assess SAV. See Figs. 7 and 8. 


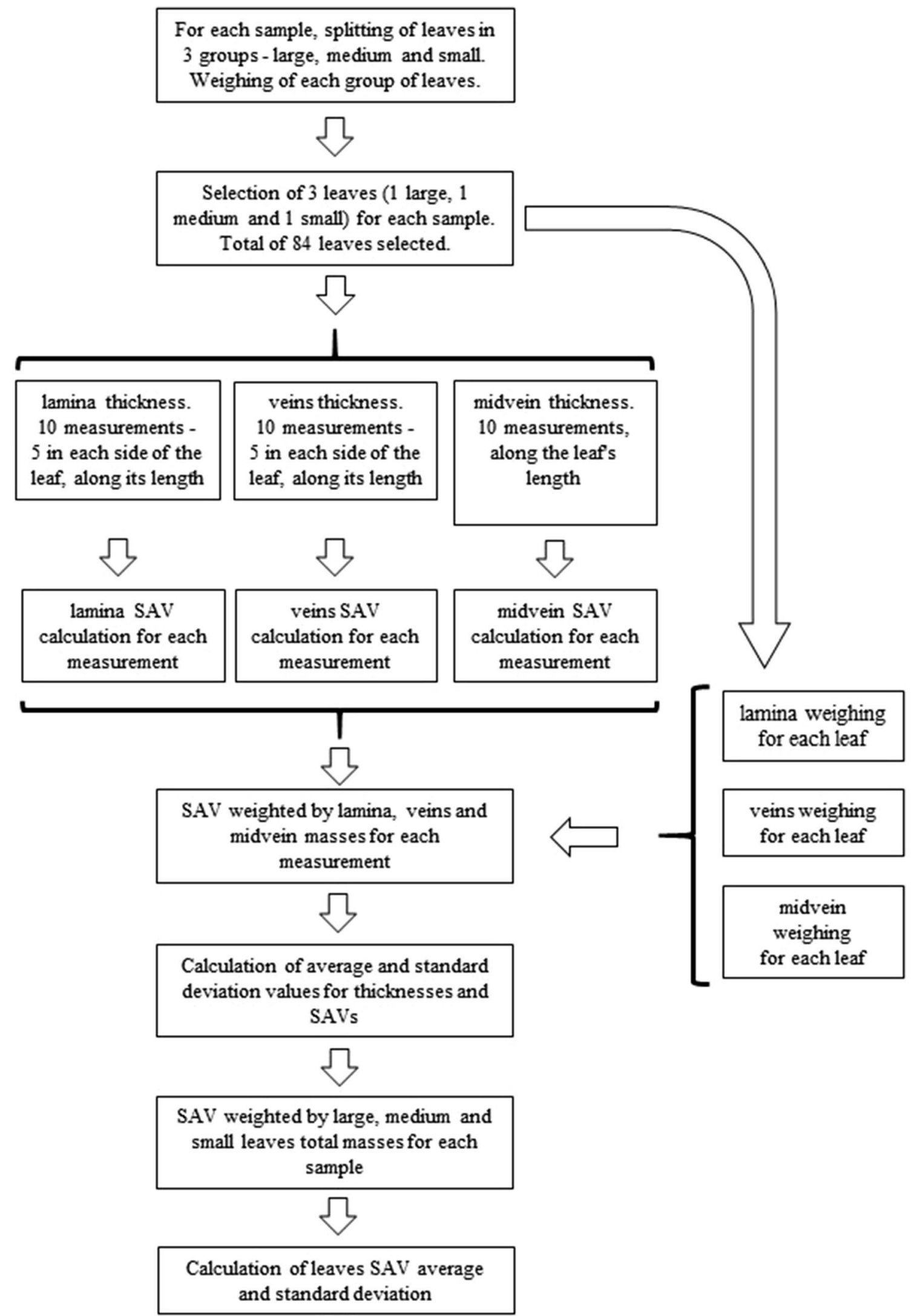

Fig. 7 Flowchart describing the procedure used to determine leaves SAV considering the midvein, veins and lamina individual SAV 
For each sample, splitting of twigs in 3 groups-large, medium and small diameter twigs.

Weighing of each group.

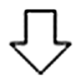

Selection of 1 specimen from each group (1 large, 1 medium and 1 small diameter twig).

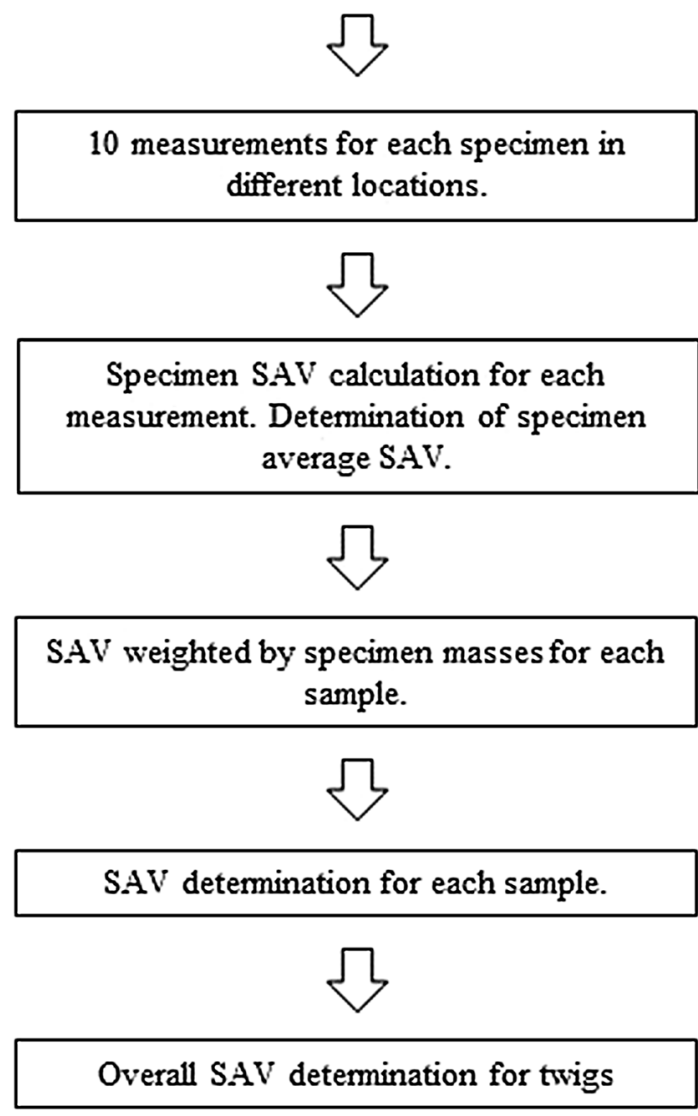

Fig. 8 Flowchart describing the procedure used to determine twigs SAV

\section{References}

1. Vogel S (1998) Life's devices: the physical world of animals and plants. Princeton University Press, Princeton, pp 38-42

2. Fernandes PM, Rego FC (1998) A new method to estimate fuel surface area to volume ratio using water immersion. Int J Wildland Fire 8:121-128

3. Papió C, Trabaud L (1990) Structural characteristics of fuel components of five Mediterranean shrubs. For Ecol Manag $35: 249-259$
4. Engber EA, Varner JM (2012) Patterns of flammability of the California oaks: the role of leaf traits. Can J For Res 42:1965-1975. https://doi.org/10.1139/x2012-138

5. Rothermel RC, Anderson HE (1966) Fire spread characteristics determined in the laboratory. USDA Forest Service, Intermountain Forest and Range Experiment Station, Research Paper INT30, Ogden, UT

6. Rundel PW (1981) Structural and chemical components of flammability. In: Mooney HA, Bonnicksen TM, Christensen NL, Lotan JE, Reiners WE (eds) Proceedings of the conference on fire regimes and ecosystems properties. USDA Forest Service, General Technical Report WO-26, Honolulu, HI, pp 183-207

7. Ottmar RD (2014) Wildland fire emissions, carbon, and climate: modeling fuel consumption. For Ecol Manag 317:41-50

8. Rothermel RC (1972) A mathematical model for predicting fire spread in wildland fuels, USDA Forest Service, Intermountain Forest and Range Experiment Station, Research Paper INT-115, Ogden, UT

9. Andrews P, Bevins CD, Seli RC (2009) BehavePlus fire modeling system, version 5.0: user's guide. USDA Forest Service, Intermountain Forest Rocky Mountain Research Station, General Technical Report RMRSGTR-106WWW, Ogden, UT

10. WFDS (2018) Web page for the WFDS model. http://www.fs.fed. us/pnw/fera/research/wfds/index.shtml. Accessed 07 Jan 2019

11. Mell WE, Maranghides A, McDermott R, Manzello S (2009) Numerical simulation and experiments of burning Douglas fir trees. Combust Flame 156:2023-2041

12. Bufacchi P, Krieger GC, Mell W, Alvarado E, Santos JC, Carvalho JA Jr (2016) Numerical simulation of surface forest fire in Brazilian Amazon. Fire Saf J 79:44-56. https://doi.org/10.1016/j.fires af.2015.11.014

13. Bufacchi P (2014) Simulação numérica de incêndios de superfície na região amazônica com modelo de turbulência de grandes estruturas. PhD Thesis, Department of Mechanical Engineering, University of São Paulo, São Paulo, Brazil

14. Sullivan AL, Surawski NC, Crawford D, Hurley RJ, Volkova L, Weston CJ, Meyer CP (2018) Effect of woody debris on the rate of spread of surface fires in forest fuels in a combustion wind tunnel. For Ecol Manag 424:236-245

15. DeLuca TH, Boisvenue C (2012) Boreal forest soil carbon: distribution, function and modelling. Forestry 85(2):161-184

16. Scott JH, Burgan RE (2005) Standard fire behavior fuel models: a comprehensive set for use with Rothermel's surface fire spread model. Gen. Tech. Rep. RMRS-GTR-153. U.S. Department of Agriculture, Forest Service, Rocky Mountain Research Station, Fort Collins, $\mathrm{CO}$

17. Brown JK (1970) Ratios of surface area to volume for common fine fuels. For Sci 16:101-105

18. Mauseth JD (2000) Theoretical aspects of surface-to-volume ratios and water-storage capacities of succulent shoots. Am J Bot 87:1107-1115

19. Hachmi M, Sesbou A, Benjelloun H, Bouanane F (2011) Alternative equations to estimate the surface-to-volume ratio of different forest fuel particles. Int J Wildland Fire 20:648-656

20. Ter Steege H, Pitman NCA, Sabatier D et al (2013) Hyperdominance in the Amazonian Tree Flora. Science 342:1243092. https ://doi.org/10.1126/science.1243092

Publisher's Note Springer Nature remains neutral with regard to jurisdictional claims in published maps and institutional affiliations. 